\title{
EFECTO DE DOS NIVELES DE NITRÓGENO Y POTASIO APLICADOS POR FERTIRRIEGO EN LAS VARIABLES DE CRECIMIENTO Y CONCENTRACIÓN DE MACRO Y MICRONUTRIENTES EN PLANTAS DE AGUACATE (Persea americana Mill.) Var. HASS
}

\author{
Gaona, Pablo ${ }^{\mathrm{a}, \mathrm{c}}$; Vásquez, Laura ${ }^{\mathrm{b}}$; Morales, Carlos ${ }^{\mathrm{b}}$; Viera, William ${ }^{\mathrm{c} *}$; Viteri, Pablo ${ }^{\mathrm{c}}$; Sotomayor, \\ Andrea $^{c}$; Medina, Lorena ${ }^{a}$; Mejía, Paúl ${ }^{d}$; Cartagena, Yamil ${ }^{c}$.

\begin{abstract}
${ }^{a}$ Instituto Interamericano de Cooperación para la Agricultura (IICA), Av. 12 de Octubre N24-584 y Francisco Salazar, Quito, Ecuador. Gasca, Quito, Ecuador.

${ }^{c}$ Instituto Nacional de Investigaciones Agropecuarias (INIAP), Av. Interoceánica km 15 y Eloy Alfaro, Tumbaco, Quito, Ecuador.

${ }^{\mathrm{d} E s p e c i a l i s t a ~ e n ~ f r u t i c u l t u r a, ~ C o n s u l t o r ~ I n d e p e n d i e n t e, ~ M a r t i ́ n ~ d e ~ A r r i o l a ~ O e 6-63, ~ L a ~ G a s c a, ~ Q u i t o, ~}$
\end{abstract} \\ ${ }^{\mathrm{b}}$ Facultad de Ciencias Agrícolas, Universidad Central del Ecuador (UCE). Jerónimo Leiton s/n y Av. La
} Ecuador.

Ingresado: $25 / 03 / 2020$

\section{Resumen}

Actualmente, el cultivo de aguacate var. Hass está tomando importancia en el Ecuador, sin embargo, existe escasa información con respecto al manejo nutricional de esta variedad, dando como resultado cosechas con baja productividad y calidad. Debido al inadecuado manejo en los ámbitos nutricional y de riego, además de la alta demanda de nitrógeno $(\mathrm{N})$ y potasio $(\mathrm{K})$ del aguacate, esta investigación tuvo como objetivo determinar el efecto de dos dosis de $\mathrm{N}$ y $\mathrm{K}$ en la fase crecimiento. El ensayo se implementó utilizando plantas de aguacate var. Hass, los tratamientos fueron aplicados mediante un sistema de fertirriego. El experimento se desarrolló en la Granja Experimental Tumbaco del INIAP bajo un diseño experimental completamente al azar (DCA) en arreglo factorial $2 \times 2$, teniendo cuatro tratamientos resultado de la interacción de dos niveles de $\mathrm{N}$ y $\mathrm{K}$ y cuatro observaciones. Se realizó la comparación de medias DMS al 5\% cuando se presentaron diferencias significativas. Las variables analizadas fueron: altura de planta, diámetro del tallo, índice de verdor, área foliar y

\footnotetext{
* Instituto Nacional de Investigaciones Agropecuarias, Av. Interoceánica km 15 y Eloy Alfaro, Quito, Ecuador. +593 999258273. Correo electrónico: william.viera@iniap.gob.ec
}

Aceptado: $11 / 06 / 2020$

concentración foliar de nutrientes. Con base a los resultados obtenidos, se observaron diferencias significativas en las variables: índice de verdor y concentración de nutrientes.

Palabras clave: Fertirriego, índice de verdor, nutrientes, raza mexicana

EFFECT OF TWO LEVELS OF NITROGEN AND POTASSIUM APPLIED BY FERTIRRIEGO IN VARIABLES OF GROWTH AND MACRO AND MICRONUTRIENT CONCENTRATION IN AVOCADO PLANTS (Persea americana Mill.) Var. HASS
Abstract
Currently, the cultivation of avocado var. Hass is becoming important in Ecuador; however, there is little information regarding the nutritional management of this crop, resulting in crops with low productivity and poor fruit quality. Due to the high demand for nitrogen $(\mathrm{N})$ and potassium $(\mathrm{K})$ of this crop and the scarce information regarding nutritional and irrigation management, this research aimed to determine the effect of two doses of $\mathrm{N}$ and $\mathrm{K}$ in the initial growth phase. The trial was implemented using avocado plants var. Hass, 
the treatments were applied using a fertigation system. The experiment was carried out in the Tumbaco Experimental Farm of INIAP, using a completely randomized design with a $2 \times 2$ factorial arrangement, having four treatments resulting from the interaction of two levels of nitrogen and potassium and four replications. The comparison of $5 \%$ DMS means was performed when there were significant differences. The variables analyzed were: plant height, stem diameter, greenery index, leaf area and foliar nutrient concentration. Based on the results obtained, significant differences were observed in the variables: greenery index and nutrient concentration.

Keywords: Fertirrigation, green index, nutrients, mexican type

\section{INTRODUCCIÓN}

En Ecuador, el cultivo de aguacate (Persea americana Mill) ha generado gran interés por parte de los productores de la sierra y costa, debido a la creciente demanda y potencial de exportación en fresco y con valor agregado. En el año 2018 se exportaron aproximadamente $126 \mathrm{t}$ de aguacate Hass con un valor de $\$ 170000$, mientras que el año 2019 las exportaciones se incrementaron alcanzando 2200 t. El Instituto Nacional de Estadísticas y Censos (INEC) reportó que en Ecuador existen aproximadamente 5579 ha de aguacate [1], concentrándose la mayor superficie en las provincias de Carchi, Imbabura, Pichincha, Tungurahua y Azuay [2]. La superficie cultivada de variedad Hass en el país es de aproximadamente 800 ha (Corpoaguacate, Reinoso, M., comunicación personal, febrero 2020). De acuerdo a la Organización de las Naciones Unidad para la Alimentación y Agricultura [3], en Ecuador en el año 2018 los rendimientos fueron de $4231 \mathrm{~kg} \mathrm{ha}^{-1}$, mientras que países vecinos como Colombia y Perú alcanzaron 7868 y $12571 \mathrm{~kg} \mathrm{ha}^{-1}$, respectivamente.

Para incrementar los rendimientos, entre los factores determinantes están el riego y la fertilización; ya que la eficiencia de absorción de cada elemento y el aporte de nutrientes requerido para la producción de $20 \mathrm{t}$ ha de aguacate son de aproximadamente $125 \mathrm{~kg}$ de N, $58 \mathrm{~kg}$ de $\mathrm{P}, 112 \mathrm{~kg}$ de $\mathrm{K}, 20 \mathrm{~kg}$ de $\mathrm{Ca}, 14 \mathrm{~kg}$ de $\mathrm{Mg}, 20 \mathrm{~kg}$ de $\mathrm{S}$ y un requerimiento hídrico entre 7500 a $10000 \mathrm{~m}^{3} \mathrm{ha}^{-1}$ año ${ }^{-1}$ [4-6]. El suministro adecuado de nutrientes y agua permitirán satisfacer los requerimientos de las plantas, tomando en consideración que, las dosis y frecuencias de aplicación deben ser balanceadas para evitar pérdidas por lixiviación y contaminación del suelo o mantos acuíferos [7]. Los bajos rendimientos y las oportunidades de exportación hacen necesario mejorar los procesos de $\mathrm{I}+\mathrm{D}+\mathrm{i}$ con el objetivo de incrementar la productividad y calidad del cultivo, además de la capacidad competitiva del país [8].

Complementariamente a lo descrito, en Ecuador el uso de fertirriego en aguacate es una tecnología poco aplicada debido a la falta de investigaciones que determinen sus beneficios en el manejo racional de agua, fertilizantes y mano de obra, y de esta manera se fomente su uso y se justifiquen los costos de implementación. Esta investigación tuvo como objetivo determinar la respuesta de la variedad Hass a la aplicación de dos niveles de $\mathrm{N}$ y K con fertirrigación y permitió definir las dosis más adecuadas para el desarrollo inicial de huertos.

\section{METODOLOGÍA}

\section{Ubicación}

La investigación se desarrolló en la Granja Experimental Tumbaco del Instituto Nacional de Investigaciones Agropecuarias (INIAP), con coordenadas: latitud $0^{\circ} 12^{\prime} 5^{\prime \prime}$ Sur, longitud $78^{\circ} 24^{\prime} 43^{\prime \prime}$ Oeste y una altitud de $2348 \mathrm{~m}$. Las precipitaciones anuales son de aproximadamente $800 \mathrm{~mm}$, concentrándose en los períodos de febrero a mayo $(400 \mathrm{~mm})$ y octubre a noviembre $(200 \mathrm{~mm})$, la temperatura media es de $17^{\circ} \mathrm{C}$ y la humedad relativa promedio de $75 \%$ [9].

Previo a la implementación del ensayo, se realizaron análisis de suelo con muestras a profundidades de $0,10,0,20$ y $0,40 \mathrm{~m}$ (Laboratorio Suelos y Aguas de la Estación Experimental Santa Catalina-INIAP). Se usaron plantas de la variedad Hass injertas en patrón tipo "criollo" (raza mexicana) de 8 meses de edad con una altura promedio de $0,40 \mathrm{~m}$, el transplante se hizo en hoyos de $0,40 \mathrm{~m}$ de profundidad, con un marco de plantación de 5 x 4 m (500 árboles ha-1). Las plantas fueron afirmadas mediante un tutor vertical; además, se realizaron monitoreos constantes para identificar problemas fitosanitarios y realizar los controles necesarios. Se monitoreó la humedad volumétrica del suelo mediante 5 sensores (marca Meter, modelo $\mathrm{ECH}_{2} \mathrm{O}$ EC5) y datalogers (Fig. 1a), instalados con una separación de $10 \mathrm{~cm}$ entre ellos e intervalos de lecturas de 5 minutos. Para compensar el 
déficit hídrico aproximado de $200 \mathrm{~mm}$ al año, se proporcionaron 2 L diarios de agua por planta, ajustados de acuerdo a las condiciones climáticas (lluvias, viento, brillo solar, temperatura, etc) y requerimientos de la especie, los riegos se fraccionaron en dos ciclos ( $1 \mathrm{~L}$ en la mañana y $1 \mathrm{~L}$ en la tarde), mediante un sistema automatizado constituido por 4 bombas de 0,5 HP, 4 tanques de 500 L de capacidad, 5 filtros ( 1 principal y 4 secundarios), doble línea de riego con goteros de $1,6 \mathrm{~L} \mathrm{~h}^{-1}$ de caudal, separados 0,30 m entre goteros (Fig. 1b). Como fuentes de cada elemento nutritivo se usaron: nitrato de amonio, nitrato calcio, nitrato magnesio, nitrato potasio, sulfato de potasio, sulfato de magnesio y ácido fosfórico. La preparación de los tratamientos se realizó por disolución de los fertilizantes en un volumen inicial de 15 litros de agua, posteriormente cada tratamiento fue incorporado a tanques con aforos de 500 L (Fig. 1c), la fertiirrigación se hizo tres días por semana con ciclos de riego de 18 minutos. Las dosis de los restantes macro (excepto $\mathrm{N}$ y $\mathrm{K}) \mathrm{y}$ microelementos correspondieron a los requerimientos reportados para este cultivo ajustados según análisis de suelo y etapa de desarrollo; además las dosis permanecieron fijas en todos los tratamientos (P, $80 \mathrm{~kg} \mathrm{ha}^{-1} ; \mathrm{Ca}$, $70 \mathrm{~kg} \mathrm{ha}^{-1} ; \mathrm{Mg}, 40 \mathrm{~kg} \mathrm{ha}^{-1} ; \mathrm{S}, 30 \mathrm{Kg} \mathrm{ha}^{-1} ; \mathrm{Fe}, 803 \mathrm{~g}$ ha-1; B, $204 \mathrm{~g} \mathrm{ha}^{-1}$; Mn, $438 \mathrm{~kg} \mathrm{ha}^{-1} ; \mathrm{Zn}, 102 \mathrm{~g} \mathrm{ha}^{-1}$ y Cu, $\left.68 \mathrm{~g} \mathrm{ha}^{-1}\right)[4,10]$.

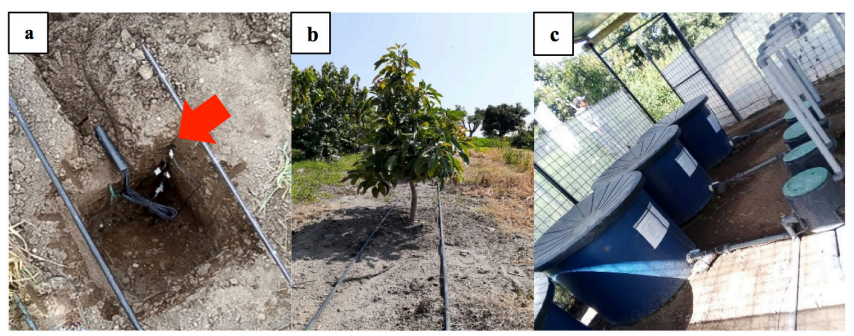

Fig. 1: Implementación del ensayo para la evaluación del efecto de 2 dosis de $\mathrm{N}$ y $\mathrm{K}$ en plantas de aguacate var. Hass con portainjerto "criollo" (raza mexicana). a) Instalación de sensores para registrar la humedad volumétrica del suelo, b) líneas de fertirriego y c) tanques de 500 L para la preparación de la solución de fertirriego.

Los factores en estudio fueron seleccionados en base a las necesidades nutricionales propias del aguacate (alta demanda de $\mathrm{N}$ y K) y a la disponibilidad de los elementos en el suelo. Es así que, los factores seleccionados fueron: a) dos elementos minerales: nitrógeno $(\mathrm{N})$ y potasio (K) y b) dos dosis de cada elemento, 200 (n1) y 300 (n2) kg ha-1 en el caso del N y 250 (k1) y 350 (k2) $\mathrm{kg} \mathrm{ha}^{-1}$ en el caso del K. Los tratamientos resultaron de la interacción de los factores en estudio con sus respectivos niveles (Tabla 1). El ensayo constó de 16 unidades experimentales, cada unidad experimental fue conformada por 9 plantas de las cuales se tomaron 3 plantas al azar para el registro de datos (se excluyeron plantas bordes), obteniendo un total de 36 plantas por tratamiento y 144 plantas en total. Se utilizó un diseño completamente al azar (DCA) con arreglo factorial 2 × 2 con cuatro tratamientos y cuatro observaciones por tratamiento. El análisis estadístico se realizó mediante un análisis de varianza, para determinar diferencias entre tratamientos se utilizó la prueba de comparación de medias DMS (5\%) y se empleó el software estadístico Infostat versión 2017 [11].

Tabla 1. Tratamientos producto de la interacción de 2 dosis de $\mathrm{N}$ y K ( $\left.\mathrm{kg} \mathrm{ha}^{-1}\right)$ aplicados por fertirriego en plantas de aguacate var. Hass con portainjerto "criollo" (raza mexicana).

\begin{tabular}{|c|c|c|}
\hline Tratamientos & $\mathbf{N}^{*}$ & $\mathbf{K}^{*}$ \\
\hline $\mathrm{n} 1 \times \mathrm{k} 1$ & 200 & 250 \\
\hline $\mathrm{n} 1 \times \mathrm{k} 2$ & 200 & 350 \\
\hline $\mathrm{n} 2 \times \mathrm{k} 1$ & 300 & 250 \\
\hline $\mathrm{n} 2 \times \mathrm{k} 2$ & 300 & 350 \\
\hline${ }^{*}\left(\mathrm{~kg} \mathrm{ha}^{-1}\right)$ & & \\
\hline
\end{tabular}

Durante 9 meses se registraron varios datos en el ensayo. La altura de planta $(\mathrm{cm})$ se evaluó a partir de 80 hasta 230 días después del trasplante $(\mathrm{ddt})$ con un intervalo de 30 días entre cada medición, las mediciones (cinta métrica) se hicieron desde el cuello de la planta hasta el ápice de la primera hoja nueva. El diámetro de tallo ( $\mathrm{mm}$ ) se evaluó en el mismo período e intervalos de tiempo que la altura de planta, las mediciones se realizaron utilizando un calibrador digital Mitutoyo modelo Vernier 500-159-30; éstas se hicieron en el portainjerto ( $5 \mathrm{~cm}$ bajo cicatriz enjertación) y en la variedad comercial ( $5 \mathrm{~cm}$ sobre cicatriz enjertación). El índice de verdor (SPAD) se evaluó en el mismo período e intervalos de tiempo que la altura de planta, se empleó un medidor de clorofila modelo SPAD-502 (Fig. 2a); en el ápice de la planta se seleccionaron 3 hojas recientemente maduras, sin color violáceo y totalmente extendidas, dichas hojas fueron remplazadas 
cada dos meses debido al envejecimiento de las hojas inicialmente seleccionadas. El área foliar $\left(\mathrm{cm}^{2}\right)$ se evaluó en el mismo período e intervalos de tiempo que la altura de planta, se usó un medidor de área foliar portátil modelo LI-COR LI3000A (Fig. 2b), fueron evaluadas las mismas hojas usadas para el índice de verdor. La concentración foliar de nutrientes (\% y

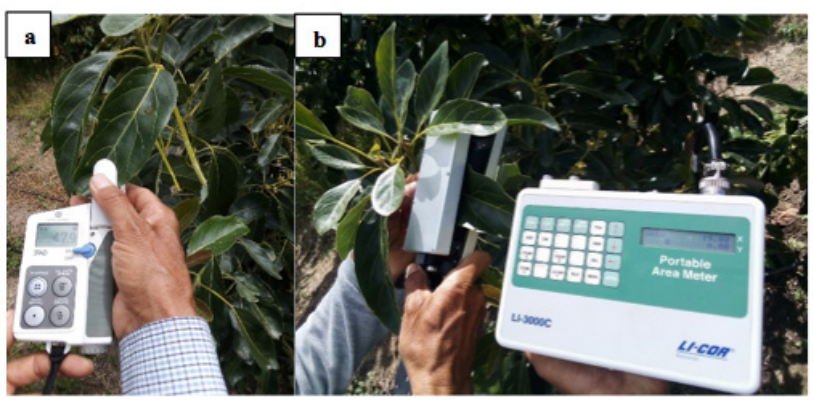

Fig. 2: Registro de las variables: a) índice de verdor y b) área foliar, en la evaluación del efecto de 2 dosis de $\mathrm{N}$ y K en plantas de aguacate var. Hass con portainjerto "criollo" (raza mexicana).

ppm) se determinó a los 180 y 270 ddt. Los macronutrientes analizados fueron nitrógeno $(\mathrm{N})$, fósforo $(\mathrm{P})$, potasio $(\mathrm{K})$, calcio $(\mathrm{Ca})$, magnesio (Mg), azufre (S); mientras que los micronutrientes fueron boro (B), zinc (Zn), cobre $(\mathrm{Cu})$, hierro $(\mathrm{Fe})$, manganeso $(\mathrm{Mn})$. Se recolectaron 150 gramos de material vegetal fresco, las muestras recolectadas se ubicaron a partir de la quinta hasta la séptima hoja de la parte apical del eje de la planta, se seleccionaron hojas de color verde ( $\sin$ color violáceo) y con el limbo totalmente extendido. Las muestras fueron enviadas al laboratorio del Departamento de Suelos y Aguas de la EESC.

\section{RESULTADOS Y DISCUSIÓN}

Una vez que las variables cumplieron los supuestos de normalidad de error y homogeneidad de varianzas, se continuó con un análisis de varianza $(\alpha \leq 0,05)$ para datos paramétricos.

\section{Altura de planta}

El análisis de varianza no reflejó diferencias significativas para los efectos simples e interacciones $\mathrm{N} \times \mathrm{K}$ durante el ensayo, se registraron rangos de alturas entre 40,92 - 45,12 (80 ddt); 47,06 - 50,27 (110 ddt); $55,27-58,12$ (140 ddt); 55, $82-60,83$ (170 ddt); 57,18 - 65,49 (200 ddt) y 67,67 - 77,19
(230 ddt) para cada período de evaluación. La ausencia de diferencias significativas entre tratamientos en fases iniciales de crecimiento concuerda con los resultados difundidos por Maldonado [12] en los que recién a partir de 2 años de realizadas a plicaciones por fertirriego de dos fuentes de $\mathrm{N}$ y $\mathrm{K}$ se evidenciaron respuestas en el crecimiento de plantas de aguacate Hass. Los rangos de fertilización reportados para $\mathrm{N}$ y K en aguacate son amplios, en el caso del $\mathrm{N}$ varía entre 125 y $300 \mathrm{~kg} \mathrm{ha}^{-1}$, mientras que en $\mathrm{K}$ son de 200 a $500 \mathrm{~kg} \mathrm{ha}^{-1}$; sin embargo, no se han reportado diferencias entre tratamientos al evaluar la influencia de dosis semejantes en el desarrollo vegetativo $[13,14]$ tomando mayor relevancia la época de aporte del nutriente que el incremento de dosis en el caso de plantas en plena producción [15]. De la misma manera, el poco efecto de las fertilizaciones edáficas en el crecimiento del cultivo en los primeros años, se debe a que la planta posee un sistema radicular con escasa capacidad de exploración (raíces $<2 \mathrm{~mm}$, ubicados en los $20 \mathrm{~cm}$ superiores del suelo) lo que ocasiona que la tasa de absorción de nutrientes sea baja [16].

\section{Diámetro del tallo}

Los tratamientos no tuvieron diámetros estadísticamente distintos para los elementos simples e interacciones $\mathrm{N} \times \mathrm{K}$, los rangos de diámetros variaron entre 9,33 - 10,88 (80 $\mathrm{ddt}) ; 9,69-11,33(110 \mathrm{ddt}) ; 9,65-11,19$ $(140 \mathrm{ddt}) ; 10,81-12,19(170 \mathrm{ddt}) ; 12,04$ $-14,20$ (200 ddt) y 12,69 - 15,65 cm (230 $\mathrm{ddt}$ ) en los distintos periodos evaluados. De manera general en especies frutales el incremento en el diámetro del tallo es lento, debido a que el $\mathrm{N}$ independiente al modo de aplicación, mantiene niveles bajos de citoquininas, mismas que se encuentran relacionadas a procesos de multiplicación celular (crecimiento de tejidos), lo cual explicaría la baja influencia de los elementos evaluados en esta variable [17]. Sin embargo, Fassio et al. reportaron incrementos en el diámetro empleando portainjertos como Toro Canyon y Duke 7 al usar soluciones nutritivas del tipo Hoagland (210 ppm de N y 235 ppm de K) [18].

\section{Índice de verdor}

Existieron diferencias significativas a los $80 \mathrm{y}$ 
170 ddt para la interacción N x K, destacándose el tratamiento $\mathrm{n} 2 \times \mathrm{k} 2$ en el primer período, mientras que los tratamientos $\mathrm{n} 1 \mathrm{x} \mathrm{k} 2 \mathrm{y} \mathrm{n} 2$ $\mathrm{x}$ k1 sobresalieron en el cuarto periodo evaluado (Tabla 2), lo que permitió visualizar de manera general que en los tratamientos con dosis altas de $\mathrm{N}$ o de $\mathrm{K}$ se dieron incrementos en los valores SPAD, frente a los tratamientos con dosis bajas de ambos elementos. Estos resultados concuerdan con reportes del índice de verdor en hojas de aguacate con mayores dosis de fertilización nitrogenada al suelo, además del efecto sinérgico del $\mathrm{K}$ en el incremento del nitrógeno foliar [4]. Aunque no se registraron diferencias significativas entre tratamientos en los monitoreos realizados a los 110 y $200 \mathrm{ddt}$, estos períodos registraron mayor actividad fotosintética en relación al resto de monitoreos, destacándose numéricamente las interacciones $\mathrm{n} 2 \mathrm{x} \mathrm{k} 2$ (54,99 SPAD) a los $110 \mathrm{ddt}$ y n2 x k1 (57,29 SPAD) a los $200 \mathrm{ddt}$, debido posiblemente al estado de plena madurez de hojas en esos periodos de evaluación; mientras que a los 140 y $230 \mathrm{ddt}$ el índice de verdor se redujo debido posiblemente a que las hojas entraron en un proceso de senescencia. La actividad fotosintética se incrementa paulatinamente hasta la plena madurez de las hojas y decae al llegar su envejecimiento, lo cual está ligado a las tasas de intercambio gaseoso relacionadas con asimilación fotosintética del $\mathrm{CO}_{2}$ y la transpiración [19], del mismo modo después de aproximadamente 30 días de brotación, las hojas de aguacate se han expandido completamente y tienen una asimilación neta de $\mathrm{CO}_{2}$ positiva [20], pero la máxima asimilación neta de $\mathrm{CO}_{2}$ se alcanza entre 40 y 70 días posteriores a la brotación, lo que explicaría el descenso fotosintético entre los periodos monitoreados en este estudio.

Tabla 2. Efectos en índice de verdor de los tratamientos evaluados ( 2 dosis de $\mathrm{N}$ y $\mathrm{K}$ ) en plantas de aguacate var. Hass con portainjerto "criollo" (raza mexicana). Prueba DMS al 5\%, letras distintas en el mismo período (columna) señalan diferencias entre tratamientos. $\mathrm{ddt}=$ días después del trasplante.

\begin{tabular}{|c|c|c|c|c|c|c|}
\hline Tratamientos & $\mathbf{8 0 ~ d d t}$ & $\mathbf{1 1 0}$ ddt & $\mathbf{1 4 0}$ ddt & $\mathbf{1 7 0 ~ d d t}$ & $\mathbf{2 0 0}$ ddt & 230 ddt \\
\hline $\mathrm{n} 1$ x k1 & $35,03 \mathrm{ab}$ & 51,19 & 38,22 & $50,17 \mathrm{~b}$ & 56,58 & 49,68 \\
\hline $\mathrm{n} 1 \times \mathrm{k} 2$ & $30,53 \mathrm{~b}$ & 53,39 & 37,77 & $57,13 \mathrm{a}$ & 53,67 & 42,26 \\
\hline $\mathrm{n} 2$ x k1 & $31,61 \mathrm{~b}$ & 53,58 & 40,30 & $58,26 \mathrm{a}$ & 57,29 & 46,00 \\
\hline $\mathrm{n} 2$ x k2 & $43,72 \mathrm{a}$ & 54,99 & 40,72 & $56,07 \mathrm{ab}$ & 53,48 & 44,15 \\
\hline
\end{tabular}

\section{Área foliar}

El análisis de varianza detectó diferencias estadísticas únicamente en el monitoreo a los $170 \mathrm{ddt}$ en las interacciones $\mathrm{N}$ x K. La prueba de DMS al $5 \%$ (Tabla 3) estableció que las dosis más altas de $\mathrm{N}$ y $\mathrm{K}$ alcanzaron el mayor valor de área foliar. El $\mathrm{N}$ al ser importante en varias funciones de la planta, también aporta favorablemente al desarrollo tanto de hojas y tallos [21], el K es el nutriente con mayor efecto sobre la velocidad de crecimiento incidiendo principalmente sobre el área foliar ya que este nutriente cumple con mantener el equilibrio en la absorción del N, prolongando la turgencia en las hojas y facilitando el transporte en la planta [22]; además, el aporte conjunto de $\mathrm{N}$ y $\mathrm{K}$ al aguacatero incrementa la capacidad fotosintética [10]. Aunque sin diferencias significativas entre tratamientos, las áreas foliares a los 200 y $230 \mathrm{ddt}$ fueron superiores numéricamente a los periodos anteriores, además la combinación de dosis más alta de $\mathrm{N}$ y $\mathrm{K}$ destacaron al compararse con el resto de tratamientos.

Tabla 3. Efectos en área foliar de los tratamientos evaluados (2 dosis de $\mathrm{N}$ y K) en plantas de aguacate var. Hass con portainjerto "criollo" (raza mexicana). Prueba DMS al 5\%, letras distintas en el mismo período (columna) señalan diferencias entre tratamientos. $\mathrm{ddt}=$ días después del trasplante.

\begin{tabular}{|c|c|c|c|c|c|c|}
\hline Tratamientos & $80 \mathrm{ddt}$ & $110 \mathrm{ddt}$ & $140 \mathrm{ddt}$ & $170 \mathrm{ddt}$ & $200 \mathrm{ddt}$ & $230 \mathrm{ddt}$ \\
\hline $\mathrm{n} 1 \mathrm{x}$ k1 & 43,09 & 46,25 & 43,46 & $60,43 a b$ & 61,24 & 63,33 \\
\hline $\mathrm{n} 1 \mathrm{xk} 2$ & 46,14 & 49,87 & 41,62 & $57,75 \mathrm{~b}$ & 65,78 & 59,10 \\
\hline $\mathrm{n} 2 \mathrm{x}$ k1 & 41,62 & 42,35 & 53,37 & $69,13 \mathrm{ab}$ & 72,38 & 73,64 \\
\hline $\mathrm{n} 2 \times \mathrm{k} 2$ & 49,66 & 54,01 & 48,09 & $70,33 a$ & 76,36 & 78,03 \\
\hline
\end{tabular}

\section{Concentración de nutrientes}

A los $180 \mathrm{ddt}$ las interacciones $\mathrm{N} x \mathrm{~K}$ presentaron diferencias significativas entre tratamientos en las concentraciones de $\mathrm{K}, \mathrm{Ca}, \mathrm{Mg}$, B y Fe; mientras que a los $270 \mathrm{ddt}$ existieron diferencias en las concentraciones de K, B, Zn y Mn (Tabla 4). El tratamiento con mayores dosis de $\mathrm{N}$ y $\mathrm{K}$ tuvo las concentraciones foliares más altas de $\mathrm{K}(2,85 \%$ y $3,27 \%$ ) a los 180 y $270 \mathrm{ddt}$, mientras que el resto de tratamientos tuvieron los valores más bajos y sin diferencias estadísticas entre sí, a excepción del tratamiento $\mathrm{n} 1 \mathrm{x} \mathrm{k} 1$ (270 ddt) que fue inferior al resto (Tabla 4). El sinergismo entre los elementos $\mathrm{N}$ y $\mathrm{K}$ se evidenció al comparar los tratamientos con dosis altas de $\mathrm{K}$ pero con diferentes niveles de $\mathrm{N}$, encontrándose 
una mayor concentración de $\mathrm{K}$ en el tratamiento con la dosis más alta de N (Tabla 4). Reportes de parcelas fertirrigadas presentaron una relación positiva entre $\mathrm{N}$ y $\mathrm{K}\left(\mathrm{R}^{2}=0,73, \mathrm{P}<0,05\right)$ [19], comprobándose una alta correlación entre estos elementos. Es importante destacar que, en aguacate la absorción y concentración de $\mathrm{N}$ en hojas es mayor a la de $\mathrm{K}$, presentando una relación aproximada de $2: 1,5$, lo cual guarda relación con la investigación llevada a cabo por Guerrero-Polanco [19] en que la relación es de 2:1. En el caso de los elementos Ca y $\mathrm{Mg}$ se registraron diferencias significativas solo a los $180 \mathrm{ddt}$, y las concentraciones de estos elementos tuvieron un comportamiento inverso con las aplicaciones de altas dosis de K. Los elementos nutritivos tanto en suelo como en tejido vegetal tienen una gran cantidad de interacciones que pueden afectar el contenido específico de cada elemento [23], con respecto a las relaciones $\mathrm{K} / \mathrm{Mg}$ y $\mathrm{K} / \mathrm{Ca}$ se han reportado fuertes antagonismos, llegándose a causar deficiencias de $\mathrm{Mg}$ y $\mathrm{Ca}$ debidas a excesos en fertilización potásica [24, 25]. La concentración de nutrientes en tejido a los $270 \mathrm{ddt}$ no tuvo diferencias estadísticas entre tratamientos para los elementos (valor promedio): $\mathrm{N}(2,91 \%), \mathrm{P}$ $(0,34 \%)$, Ca $(1,92 \%), \mathrm{Mg}(0,58 \%), \mathrm{S}(0,43 \%)$, $\mathrm{Cu}(6,58 \mathrm{ppm})$ y Fe $(124,60 \mathrm{ppm}) ;$ en el caso del $\mathrm{N}$ la concentración detectada en el presente trabajo fue superior a los rangos reportados para la variedad Hass por Salvo y Lovatt $(2,28 \%$ a $2,62 \%)$ [15], Lazare et al. $(1,41 \%$ a 1,46\%) [26] y Sotomayor et al. (1,68\% a $1,79 \%$ ) [27], el contenido de $\mathrm{P}$ fue mayor al compararlo con los reportes de Selladurai y Awachare $(0,1 \%$ a $0,25 \%)$ y [28] Sotomayor et al. $(0,065$ a $0,07 \%)$ [27], mientras que las concentraciones de Ca fueron superiores a los rangos 1,55\% y 1,63 reportados por Lazare et al [26] y estuvieron dentro del rango (1\% a $2,1 \%)$ reportado por Silber et al [13]. El resto de macro y micronutrientes (excepto el B y $\mathrm{Zn)}$ se mantuvo en estándares normales según reportes bibliográficos [4, 15 y 26].

A los $270 \mathrm{ddt}$, el contenido de B en el tratamiento $\mathrm{n} 1 \mathrm{x} \mathrm{k} 1$ fue superior al resto de tratamientos (Tabla 4), valor que excede a los reportados por Tzatzani et al. (24 a 26 ppm) [29], Martínez et al. (40 a 60 ppm) [4], pero están dentro de los rangos encontrados por Lazare et al. y Lahav y Kadman (114 a 121 ppm). [26, 29] En suelos con pH bajo, la disponibilidad y absorción de este elemento se eleva y puede causar toxicidad [4, 29], que se manifiesta en síntomas característicos como quemadura de hojas, defoliación y menor contenido de clorofila $[29,30]$. En el caso del $\mathrm{Zn}$, todos los tratamientos excepto $\mathrm{n} 2 \mathrm{x} \mathrm{k} 2$ obtuvieron concentraciones similares a los $270 \mathrm{ddt}$, estos valores se aproximaron al límite máximo reportado para este catión [30, 32]. El exceso de Zn ocasiona inhibición drástica en el desarrollo de la planta (tallo y raíces), además de causar clorosis en hojas [32]. A lo largo del estudio no se evidenciaron síntomas de excesos de B y Zn en las hojas, además las plantas mostraron valores SPAD y de área foliar dentro de los rangos normales para la especie [31].

Tabla 4. Efectos en la concentración de macro y micronutrientes en hojas producto de los tratamientos evaluados (2 dosis de $\mathrm{N}$ y K) en plantas de aguacate var. Hass con portainjerto "criollo" (raza mexicana). Prueba DMS al 5\%, letras distintas en el mismo período (columna) señalan diferencias entre tratamientos. ddt = días después del trasplante.

\begin{tabular}{|c|c|c|c|c|c|c|c|c|c|c|c|c|}
\hline \multirow{2}{*}{ ddt } & \multirow{2}{*}{ Tratamientos } & \multicolumn{6}{|c|}{ Macronutrientes (\%) } & \multicolumn{5}{|c|}{ Micronutrientes (ppm) } \\
\hline & & $\mathbf{N}$ & $\mathbf{K}$ & $\mathbf{P}$ & $\mathbf{C a}$ & Mg & $\mathbf{S}$ & B & $\mathbf{Z n}$ & $\mathbf{C u}$ & $\mathbf{F e}$ & Mn \\
\hline \multirow{4}{*}{180} & $\mathrm{n} 1 \mathrm{x} \mathrm{k1}$ & 3,71 & $1.75 \mathrm{~b}$ & 0,26 & $1,57 \mathrm{a}$ & $0,50 \mathrm{a}$ & 0,33 & $34,38 \mathrm{ab}$ & 24,75 & 8,78 & $138,43 \mathrm{ab}$ & 114,08 \\
\hline & $\mathrm{n} 1 \times \mathrm{k} 2$ & 3,61 & $1,79 \mathrm{~b}$ & 0,28 & $1,45 \mathrm{ab}$ & $0,45 \mathrm{ab}$ & 0,33 & $29,58 \mathrm{bc}$ & 27,08 & 8,23 & $105,25 \mathrm{~b}$ & 107,50 \\
\hline & $\mathrm{n} 2 \times \mathrm{k} 1$ & 3,64 & $1,82 \mathrm{~b}$ & 0,26 & $1,51 \mathrm{ab}$ & $0,47 \mathrm{ab}$ & 0,34 & $27,28 \mathrm{c}$ & 24,38 & 8,33 & $112,13 \mathrm{~b}$ & 152,93 \\
\hline & $\mathrm{n} 2 \times \mathrm{k} 2$ & 3,47 & $2,85 \mathrm{a}$ & 0,28 & $1,24 \mathrm{~b}$ & $0,43 \mathrm{~b}$ & 0,35 & $36,43 \mathrm{a}$ & 29,95 & 7,68 & $153,08 \mathrm{a}$ & 127,55 \\
\hline \multirow{4}{*}{270} & $\mathrm{n} 1 \times \mathrm{k} 1$ & 2,89 & $2,08 \mathrm{c}$ & 0,36 & 1,80 & 0,57 & 0,44 & $111,10 \mathrm{a}$ & $187,75 \mathrm{a}$ & 5,18 & 132,5 & $126,03 \mathrm{~b}$ \\
\hline & $\mathrm{n} 1 \times \mathrm{k} 2$ & 2,91 & $2,35 \mathrm{~b}$ & 0,31 & 1,98 & 0,57 & 0,40 & $54,85 \mathrm{~b}$ & $194,30 \mathrm{a}$ & 6,93 & 124,53 & $150,08 \mathrm{~b}$ \\
\hline & $\mathrm{n} 2 \times \mathrm{k} 1$ & 2,77 & $2,37 \mathrm{~b}$ & 0,33 & 2,28 & 0,64 & 0,42 & $52,98 \mathrm{~b}$ & $199,53 \mathrm{a}$ & 7,30 & 124,15 & 291,03 a \\
\hline & $\mathrm{n} 2 \times \mathrm{k} 2$ & 3,05 & $3,27 \mathrm{a}$ & 0,36 & 1,63 & 0,53 & 0,46 & $80,40 \mathrm{~b}$ & $132,40 \mathrm{~b}$ & 6,90 & 117,23 & $155,70 \mathrm{~b}$ \\
\hline
\end{tabular}




\section{CONCLUSIONES Y RECOMENDACIONES}

Las dosis altas de $\mathrm{N}$ y $\mathrm{K}$ (300 y $350 \mathrm{~kg} \mathrm{ha}^{-1}$ ) aplicadas en la fase inicial de desarrollo vegetativo, influyeron positivamente en las variables de índice de verdor, área foliar y concentración de $\mathrm{K}$ foliar; consecuentemente, se evidenció el sinergismo entre el $\mathrm{N}$ y K aplicados en dosis altas, beneficiando el desarrollo de plantas en la fase de formación de huerto. El estatus nutricional de elementos en hojas estuvo dentro de parámetros óptimos reportados para esta especie, lo que garantizaría un desarrollo sostenible y acelerado de la canopia del huerto hasta llegar a etapas productivas con una buena estructuración de plantas. Sin embargo, se observó que la interacción de las dosis altas de ambos elementos influyó en la absorción de $\mathrm{K}$ y Fe; mientras que la interacción de las dosis bajas incidió en la absorción de $\mathrm{Ca}$, Mg y B. La investigación debe continuar hasta la etapa productiva para poder evaluar el impacto de los tratamientos en el rendimiento y calidad de frutos; además se deberían incluir distintas dosis de P para evaluar la incidencia de este elemento en etapas de desarrollo más avanzadas.

\section{AGRADECIMIENTOS}

Al Fondo Regional de Tecnología Agropecuaria (FONTAGRO), mediante el proyecto Productividad y Competitividad Frutícola Andina que financió esta investigación; al Instituto Interamericano de Cooperación para la Agricultura (IICA) y al Instituto Nacional de Investigaciones Agropecuarias (INIAP) que apoyaron este estudio. Al Programa Nacional de Fruticultura-INIAP y a todo el personal que labora en la Granja Experimental Tumbaco quienes apoyaron el desarrollo de esta investigación.

\section{REFERENCIAS}

[1] Instituto Nacional de Estadísticas y Censos (INEN). Encuesta de superficie y producción agropecuaria continua 2016". Quito, Ecuador; 2017

[2] Viera W, Ponce L, Morillo E y Vásquez W. Genetic Variability of Avocado Germplasm for Plant Breeding. International Journal of Clinical and Biological Sciences. 2015; 1 (1): 24-33.

[3] Organización de las Naciones Unidad para la Alimentación y Agricultura, División de Estadisticas (FAOSTAT). http://faostat3.fao.org / browse/Q/*/E." por "http://faostat3.fao.org
[4] MartínezJ, MuenaV, RuizR. Nutricióny Fertilidad en Palto. Boletín Instituto de Investigaciones Agropecuarias (INIA). 2014; 1(283):74.

[5] Moreno G, Pliego C, Sarmiento D, Barceló A, Martínez-Ferri E. Yield and fruit quality of avocado trees under different regimes of water supply in the subtropical coast of Spain. Agricultural Water Management. 2019; 221 : 192-201.

[6] Holzapfel E, De Souza JA, Jara J, Guerra HC. Responses of avocado production to variation in irrigation levels. Irrigation science. 2017; 35(3) : 205-215.

[7] Zamudio B, Mendoza E, Alcántar G, Etchevers JD, Vázquez A. Pérdida de nitratos y salinización del suelo por riego por goteo con nitrógeno. Terra Latinoamericana. 2011; 9 (3): 239-248.

[8] Viera A, Sotomayor A, Viera W. Potencial del cultivo de aguacate (Persea americana Mill) en Ecuador como alternativa de comercialización en el mercado local e internacional. Revista científica y Tecnológica USPE. 2016; 3 (3): 1-9.

[9] Instituto Nacional de Meteorología en hidrología (INAMHI). Precipitación, humedad relativa. Quito. Ecuador. 2019

[10] Whiley A, Schaffer B, Wolstenholme N. El Aguacate: Botánica, Producción y Usos. Valparaíso, Chile: Ediciones Universitarias de Valparaíso. 2015. p. 26.

[11] Di Rienzo J, Casanoves F, Balzarini M, Gonzalez L, Tablada M, Robledo C. InfoStat versión 2016, Grupo InfoStat, FCA, Universidad Nacional de Córdova, Argentina. 2016. Disponible en: http:// www.infostat.com.ar

[12] Maldonado T. Diagnóstico nutrimental para la producción de aguacate Hass (Tesis de pregrado). Universidad Autónoma Chapingo; 2002.

[13] Silber A, Naor A, Cohen H, Bar-Noy Y, Yechieli N, Levi M, Assouline S. Avocado fertilization: Matching the periodic demand for nutrients. Scientia Horticulturae. 2018; 241: 231-240.

[14] Selladurai R, Awachare CM. Nutrient management for avocado (Persea americana miller). Journal of Plant Nutrition.2020 ; 43(1) : 138-147. 
[15] Salvo JE, Lovatt CJ. Nitrogen fertilization strategies for the 'Hass' avocado that increase total yield without reducing fruit size. HortTechnology. 2016; 26(4): 426-435.

[16] Salazar S, Cossio LE, González I. La fertilización de sitio específico mejoró la productividad del aguacate Hass en huertos sin riego. Agricultura Técnica México. 2009; 35 (4): 436-445.

[17] Etchevers, J. Determinación de nitrógeno en el suelo. Sociedad mexicana de la ciencia del suelo 1. 1987. p. 45-83.

[18] Fassio C, Heath R, Arpaia M, Castro M. Sap flow in 'Hass' avocado trees on two clonal rootstocks in relation to xylem anatomy. Ciencia Horticultura. 2009; 120: 8-13.

[19] Guerrero F, Alejo G, Sánchez R, Bugarín $\mathrm{R}$, Aburto A, Isiordia N. Respuesta del cultivo de aguacate (Persea americana Mill.) variedad 'Hass' a la aplicación de nitrato de potasio. Acta Agronómica. 2018; 67 (3): 425-430.

[20] Schaffer B, Wolstenholme B, Whiley A.The Avocado: Botany, Production and Uses. 2nd ed. CAB International. Oxfordshire, UK. 2013. p. 560

[21] Bernal E, Díaz D. Tecnología para el cultivo de aguacate. Antioquía, Colombia: CORPOICA, Manual Técnico. ํ⒌ 2006.

[22] Pillimue G, Barrera N, De Cantillo S. Determinación de deficiencias de elementos mayores en plantas de tomate de árbol". Acta Agronómica. 1998; 48(3): 62-67.

[23] Robson A, Pitman MG. Interacción entre nutrientes en plantas superiores. Enciclopedia de fisiología vegetal. 1983. p. 180.

[24] Casierra F, Lizarazo L, Andrade G. Estado nutricional de árboles de manzano 'Anna' durante la estación. Agronomia Colombia. 2004; 22 (2): 160-169.

[25] Navarro G, Navarro S. Fertilizantes, química y acción. España. Editorial Mundi-Prensa. 2014

[26] Lazare S, Haberman A, Yermiyahu U, Erel R, Simenski E, Dag A. Avocado rootstock influences scion leaf mineral content. Archives of Agronomy and Soil Science. 2019; 1: 1-11.
[27] Sotomayor A, Gonzáles A, Cho KJ, Villavicencio A, Jackson T, Viera W. Effect of the application of microorganisms on the nutrient absorption in avocado (Persea americana Mill.) seedlings. The Journal of the Korean Society of International Agriculture. 2019; 31(1): 17-24.

[28] Selladurai R, Awachare CM. Nutrient management for avocado (Persea americana miller). Journal of Plant Nutrition. 2020 ; 43(1) : 138-147.

[29] Tzatzani TT, Kavroulakis N, Doupis G, Psarras G, Papadakis IE. Nutritional status of 'Hass' and 'Fuerte'avocado (Persea americana Mill.) plants subjected to high soil moisture. Journal of Plant Nutrition. 2020 ; 43(3):327-334.

[30] Lahav E, Kadman A. Avocado fertilisation No. 6. International Potash Institute. 1980

[31] Novoa MA, Miranda D, Melgarejo LM. Efecto de las deficiencias y excesos de fósforo, potasio y boro en la fisiología y el crecimiento de plantas de aguacate (Persea americana, cv. Hass). Revista Colombiana de Ciencias Hortícolas.2018; 12(2): 293-307.

[32] Medas D, De Giudici G, Pusceddu C, Casu MA, Birarda G, Vaccari L, Meneghini C. Impact of $\mathrm{Zn}$ excess on biomineralization processes in Juncus acutus grown in mine polluted sites. Journal of hazardous materials.2019; 370: 98-107. 